\title{
Approximating Euclidean Distances by Small Degree Graphs*
}

\author{
José Soares \\ Universidade de São Paulo, São Paulo, Brazil \\ jose@ime.usp.br \\ and \\ University of Chicago, Chicago, IL, USA
}

\begin{abstract}
Given an undirected edge-weighted graph $G=(V, E)$, a subgraph $G^{\prime}=$ $\left(V, E^{\prime}\right)$ is a $t$-spanner of $G$ if, for every $u, v \in V$, the weighted distance between $u$ and $v$ in $G^{\prime}$ is at most $t$ times the weighted distance between $u$ and $v$ in $G$.

We consider the problem of approximating the distances among points of a Euclidean metric space: given a finite set $V$ of points in $\mathbb{R}^{d}$, we want to construct a sparse $t$-spanner of the complete weighted graph induced by $V$. The weight of an edge in these graphs is the Euclidean distance between the endpoints of the edge.

We show by a simple greedy argument that, for any $t>1$ and any $V \subset \mathbb{R}^{d}$, a $t$-spanner $G$ of $V$ exists such that $G$ has degree bounded by a function of $d$ and $t$. The analysis of our bounded degree spanners improves over previously known upper bounds on the minimum number of edges of Euclidean $t$-spanners, even compared with spanners of bounded average degree. Our results answer two open problems, one proposed by Vaidya and the other by Keil and Gutwin.

The main result of the paper concerns the case of dimension $d=2$. It is fairly easy to see that, for some $t(t \geq 7.6), t$-spanners of maximum degree 6 exist for any set of points in the Euclidean plane, but it was not known that degree 5 would suffice. We prove that for some (fixed) $t, t$-spanners of degree 5 exist for any set of points in the plane. We do not know if 5 is the best possible upper bound on the degree.
\end{abstract}

\section{Introduction}

Let $G=(V, E)$ be an edge-weighted graph where the weight function $w$ is an arbitrary function $w: E \rightarrow \mathbb{R}^{+}$. By the length of a path $\left(x_{0}, x_{1}, \ldots, x_{k}\right)$ in $G$ we mean the sum $\sum_{i=1}^{k} w\left(x_{i-1} x_{i}\right)$. The G-distance $d_{G}(x, y)$ of $x, y \in V$ is the length of the

\footnotetext{
* This research was supported by Conselho Nacional de Desenvolvimento Cientifico e Tecnológico, Proc 203039/87.4 (Brazil).
} 
shortest path between $x$ and $y$. We say that a subgraph $G^{\prime}=\left(V, E^{\prime}\right)$ (with the same weights on $E^{\prime}$ ) is a $t$-spanner of $G$ if, for every $x, y \in V$,

$$
d_{G} \cdot(x, y) \leq t \cdot d_{G}(x, y)
$$

The number $t$ is a measure of how well $G^{\prime}$ approximates $G$ with respect to the distances. The construction of $t$-spanners has received recent attention in several works: [2], [3], [5], [8], [9], [11], and [18], among others.

Given a set $V \subset \mathbb{R}^{d}$ the complete Euclidean graph on $V$ is the complete graph on $V$ where each edge weight is the Euclidean distance $\|x-y\|$. In this paper we consider the problem of constructing bounded degree spanners of complete Euclidean graphs. For brevity we write $t$-spanner of $V$ instead of $t$-spanner of the complete Euclidean graph on $V$.

Let $\Delta(G)$ denote the maximum degree of a graph $G$. Dobkin et al. [5] mention that Feder and others had shown that, for some fixed $t$ and for any set $V$ of points in the Euclidean plane, a $t$-spanner $G$ of $V$ exists such that $\Delta(G) \leq 7$. Then they ask what would be the minimum $\Delta$ for which such a result is possible? This paper has a partial answer to this question. Our main result (Section 4) is that, for some fixed $t, t$-spanners with $\Delta \leq 5$ exist. Nisan [10] has proved the same for $\Delta \leq 6$.

Section 2 contains the basic algorithm used to construct bounded degree $t$-spanners. Although the algorithm has been used before by Althöfer et al. [1] and Soares [16] to construct $t$-spanners for arbitrary graphs, it was not known that the algorithm also constructs bounded degree spanners for complete Euclidean graphs.

Section 3 contains a brief analysis of the problem when $V$ is in $d$-dimensional Euclidean space. We show that, for any $t>1$ and any $V \subset \mathbb{R}^{d}$, a $t$-spanner $G$ of $V$ exists where $\Delta(G)$ is bounded by a function that depends only on $d$ and $t$. This answers a question proposed by Keil and Gutwin in [8]. This bound on the maximum degree implies an improvement on the previously known upper bounds on the number of edges sufficient to build Euclidean spanners. Then we show that, for each dimension $d$, the least $\Delta(G)$ for which our algorithm constructs $O_{d}(1)$ spanners coincides with the kissing number in dimension $d .\left(O_{d}(1)\right.$ denotes some function of $d$, i.e., a constant for each $d$.)

Section 4 contains our main result, the construction of $O(1)$-spanners of degree 5 for any set of points in the Euclidean plane.

\section{The Greedy Algorithm}

Henceforth, all graphs will be embedded in a Euclidean space. Accordingly, metric concepts are understood relative to this embedding. The Euclidean distance between $x$ and $y$ is denoted by $d(x, y)$.

We now present the algorithm to construct bounded degree spanners. The input of the algorithm is a set $V$ of points in a Euclidean space of dimension $d$ and a real number $t>1$. We show in Section 3 that the bound on the degree depends only on $d$ and $t$. 


\section{Greedy Algorithm}

Input: a set $V$ of points in a Euclidean space and a real number $t>1$.

Output: a $t$-spanner $G=(V, E)$ of $V$.

\section{begin}

sort the pairs $x, y \in V$ in nondecreasing order of the distances $d(x, y)$;

$E:=\varnothing ; G:=(V, E)$;

for each pair $x, y \in V$ (from the sorted list) do

$$
\text { if } \begin{aligned}
d_{G}(x, y)>t \cdot d(x, y) \text { then } \\
E:=E \cup\{x y\} ; \\
G:=(V, E) ;
\end{aligned}
$$

output $G=(V, E)$

end.

Since the algorithm inspects all possible distances among points of $V$ it is clear that $G$ is a $t$-spanner of $V$. The following proposition states that the angle formed by any two edges of $G$ incident with the same vertex is not small. This fact allows us to bound the maximum degree of $G$.

Proposition 2.1. Let $G=(V, E)$ be the output of the Greedy Algorithm on input $V$ and $t$. Then the angle formed by any two edges incident with the same vertex is larger than

$$
\alpha=2 \arcsin \frac{t-1}{2 t} .
$$

Before proving this proposition we prove a stronger property concerning the angle formed by edges in $G$. We need this property in Section 4.

Proposition 2.2. Let $G=(V, E)$ be the output of the Greedy Algorithm on input $V$ and $t$. Let $x, y, z \in V$ be such that $x y, x z \in E$ (see Fig. 1). Let $r=d(x, z) / d(x, y)$, $\gamma=\angle y x z$. Then

$$
\cos \gamma<\frac{1}{2 r}\left(1-\frac{1}{t^{2}}\right)+\frac{1}{t}
$$

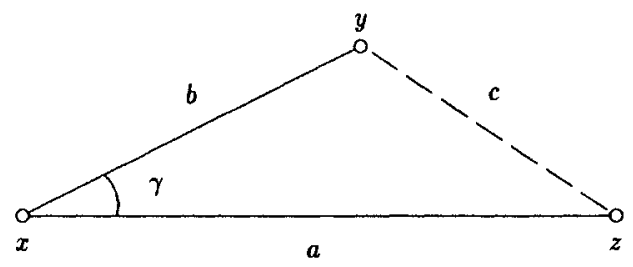

Fig. 1. Illustration for Proposition 2.2. 
Proof. Let $a=d(x, z), b=d(x, y)$, and $c=d(y, z)$. Since the value of the expression $(1 / 2 r)\left(1-1 / t^{2}\right)+1 / t$ decreases when $r=a / b$ increases, it is enough to prove the proposition for $a \geq b$. From now on we assume that $a \geq b$ and $r \geq 1$.

By a basic geometric fact we have that

$$
c^{2}=a^{2}+b^{2}-2 a b \cos \gamma
$$

First we suppose that $a \leq c$. Then

$$
a^{2} \leq c^{2}=a^{2}+b^{2}-2 a b \cos \gamma
$$

and we have that

$$
\cos \gamma \leq \frac{b}{2 a}=\frac{1}{2 r}<\frac{1}{2 r}+\frac{2 r t-1}{2 r t^{2}}=\frac{1}{2 r}\left(1-\frac{1}{t^{2}}\right)+\frac{1}{t},
$$

as desired.

Now, we suppose that $a>c$. This implies that the pair $y, z$ was inspected before the pair $x, z$ by the Greedy Algorithm. We infer that in the partially constructed graph $G$ a $y z$-path existed that did not use the edge $x z$ and had length at most $t c$. Since $a \geq b$, we may assume without loss of generality that the pair $x, y$ comes before the pair $x, z$ in the ordering of pairs considered by the Greedy Algorithm. Thus we also infer that the edge $x y$ is present in the partially constructed graph at the time the pair $x, z$ was being inspected. Since $x z$ was added to $E$, we deduce that $b+t c>t a$, or

$$
c>a-\frac{b}{t}
$$

From (2) and (1) we obtain

$$
a^{2}+b^{2}-2 a b \cos \gamma>\left(a-\frac{b}{t}\right)^{2} \quad \text { or } \quad \cos \gamma<\frac{1}{2 r}\left(1-\frac{1}{t^{2}}\right)+\frac{1}{t}
$$

as desired.

Proof of Proposition 2.1. Let $x, y, z \in V$ be such that $x y, x z \in E, a=d(x, z)$, $b=d(x, y), c=d(y, z), r=a / b$, and $\gamma=\angle y x z$ (see Fig. 1). Without loss of generality assume that the pair $x, y$ comes before the pair $x, z$ in the ordering considered by the Greedy Algorithm $(d(x, y) \leq d(x, z))$. We know from Proposition 2.2 that

$$
\cos \gamma<\frac{1}{2 r}\left(1-\frac{1}{t^{2}}\right)+\frac{1}{t}
$$

We want to prove that $\gamma>\alpha=2 \arcsin ((t-1) / 2 t)$. 
Since $d(x, y) \leq d(x, z)$ we have that $r \geq 1$. This implies that

$$
\cos \gamma<\frac{1}{2 r}\left(1-\frac{1}{t^{2}}\right)+\frac{1}{t} \leq \frac{1}{2}\left(1-\frac{1}{t^{2}}\right)+\frac{1}{t}
$$

Substituting $t=1 /(1-2 \sin (\alpha / 2))$ in the expression above we obtain

$$
\cos \gamma<\frac{1}{2}\left(1-\left(1-2 \sin \left(\frac{\alpha}{2}\right)\right)^{2}\right)+1-2 \sin \left(\frac{\alpha}{2}\right)=1-2 \sin ^{2}\left(\frac{\alpha}{2}\right)=\cos \alpha,
$$

implying that $\gamma>\alpha$, as desired.

\section{The Results for the $d$-Dimensional Case}

Let $A(d, \alpha)$ be the maximum number of rays (half-lines) from a point in $d$ dimensional Euclidean space such that each pair of rays forms an angle at least $\alpha$. It follows from Proposition 2.1 that $A(d, \alpha)$ is an upper bound on the degree of the $t$-spanner constructed by the Greedy Algorithm, where $\alpha=2 \arcsin ((t-1) / 2 t)$.

Let $\sigma_{d}$ denote the unit sphere with center $v$ in $\mathbb{R}^{d}$. A finite set of points on $\sigma_{d}$ is called a spherical code. It is easy to see that $A(d, \alpha)$ is the maximum cardinality of a spherical code $V$ such that $\angle x v y \geq \alpha$ for each $x \neq y \in V$. This packing problem has been extensively studied [4]. We mention an upper bound due to Rankin [12]:

$$
A(d, \alpha) \leq\left(\frac{1}{2} \pi d^{3} \cos \alpha\right)^{1 / 2}\left(\sqrt{2} \sin \left(\frac{\alpha}{2}\right)\right)^{-d}(1+o(1)) .
$$

(Other known bounds are stronger than this for certain ranges of values of $(d, \alpha)$.)

Since in our case $\sin (\alpha / 2)=(t-1) / 2 t$, we obtain the following theorem.

Theorem 3.1. Given a set $V$ of points in the d-dimensional Euclidean space and a real number $t>1$, a t-spanner $G=(V, E)$ of $V$ exists such that

$$
\Delta(G)=O\left(d^{3 / 2}\left(\frac{\sqrt{2} \cdot t}{t-1}\right)^{d}\right)
$$

For dimension 2 , we have that $A(2, \alpha)=\lfloor 2 \pi / \alpha\rfloor$. It follows that:

Corollary 3.2. Given a set $V$ of points in the Euclidean plane and a real number $t>1$, a $t$-spanner $G=(V, E)$ of $V$ exists such that

$$
\Delta(G) \leq\left\lfloor\frac{2 \pi}{\alpha}\right\rfloor
$$

where $\alpha=2 \arcsin ((t-1) / 2 t)$. 


\subsection{Comparison with Previous Results}

The algorithms discovered by Salowe [14] and Vaidya [18] to build $t$-spanners are similar to each other. Both are based on Vaidya's algorithm to solve the all-nearest-neighbor problem [17]. Also both require that $1<t \leq 2$. Salowe's construction leads to a $t$-spanner with $O\left((16 d t /(t-1))^{d} n\right)$ edges, while Vaidya's requires $O\left(2^{d}(3+12 \sqrt{d} /(t-1))^{d} n\right)$ edges. Vaidya has asked [18] whether the superexponential growth can be reduced as a function of the dimension $d$. Our bound answers this question in the affirmative.

We have shown that the bound on the degree of the $t$-spanner constructed by the Greedy Algorithm is related to a sphere-packing problem. Other schemes obtain spanners whose bound on the number of edges is related to a spherecovering problem. That is the case for the algorithms described by Althöfer et al. [2] and Ruppert and Seidel [13], where the bound on the number of edges is $O\left(d^{3 / 2} \log d \cdot(2 \cdot t /(t-1))^{d} \cdot n\right)$.

We remark that none of these algorithms construct bounded degree spanners for fixed dimension; only their average degree is bounded.

\subsection{An Upper Bound on $\Delta(G)$}

We now analyze the least $\Delta(G)$ for which the Greedy Algorithm outputs an $O_{d}(1)$-spanner. The function $A(d, \alpha)$ gives us a bound on $\Delta(G)$ that depends on $d$ and $\alpha$. Since this function decreases when $\alpha$ increases, we want to choose a large value for $\alpha$. However, $\alpha=2 \arcsin ((t-1) / 2 t)$, hence $\alpha<\pi / 3$. So, the smallest value of $\Delta(G)$ cannot be less than $A(d, \pi / 3)$. On the other hand, for each $d \geq 2$ a positive constant $\delta_{d}$ exists such that $A(d, \pi / 3)=A\left(d, \pi / 3-\delta_{d}\right)$. Since for large values of $t$ we can make $\alpha$ arbitrarily close to $\pi / 3$, we have the following proposition:

Proposition 3.3. Given a set $V$ of points in d-dimensional Euclidean space, a $t$-spanner $G=(V, E)$ of $V$ exists such that $\Delta(G) \leq A(d, \pi / 3)$, and

$$
t \leq \frac{1}{1-2 \sin \left(\left(\pi / 3-\delta_{d}\right) / 2\right)}
$$

The number $A(d, \pi / 3)$ is known as the kissing number: the maximum number of spheres that can touch one sphere, all spheres having the same radius. The following upper bound for $A(d, \pi / 3)$ is from [6]:

$$
A\left(d, \frac{\pi}{3}\right) \leq 2^{0.401 d(1+o(1))}
$$

Some values of $A(d, \pi / 3)$ are: $6,12,24-25,40-46,72-82,126-140,240$, corresponding to $d=2,3, \ldots, 8$, respectively. A range indicates that the exact value 
for $A(d, \pi / 3)$ is not known. These numbers were compiled by Conway and Sloane [4], a good reference for both spherical codes and kissing numbers.

For dimension 2, we may apply Proposition 3.3 using $\pi / 3-\delta_{2}=0.898$ to obtain the following:

Corollary 3.4. Every set of points in the Euclidean plane has a 7.6-spanner with maximum degree at most 6.

\section{The Main Result}

Corollary 3.4 shows that degree 6 graphs suffice to approximate planar distances within a 7.6 factor. It is much more difficult to obtain a reasonable approximation with maximum degree $\Delta(G) \leq 5$. This section is dedicated to the proof that this is possible. The main theorem is a direct consequence of Proposition 4.8(v).

Theorem 4.1 (Main Theorem). Every set of points in the Euclidean plane has a $\bar{t}$-spanner with maximum degree at most 5 , where $\bar{t}$ is an absolute constant.

We briefly describe the strategy to construct $G$, the $\bar{t}$-spanner of the given set $V \subset \mathbb{R}^{2}$. Our analysis showed that, for a convenient $t \geq 7.6$, the Greedy Algorithm outputs a $t$-spanner of maximum degree at most 6 . Indeed, there are sets of points in the plane for which degree 6 will actually occur. We first characterize such configurations, which we call bad configurations. The algorithm to build the $i$-spanner $G$ with $\Delta(G) \leq 5$ will carefully remove points from the given set $V$ (destroying the bad configurations), obtaining a set $V^{\prime}$ for which the Greedy Algorithm builds a $t^{\prime}$-spanner $G^{\prime}$ with maximum degree at most 5 . Then we show how to construct the spanner $G$ from $G^{\prime}$ by connecting the points in $V \backslash V^{\prime}$ to points in $V^{\prime}$, adding or removing edges if necessary.

\subsection{Characterizing a Bad Configuration}

We want to characterize the configurations which cause the Greedy Algorithm to output a $t$-spanner with maximum degree equal to 6 . Consider a $t$-spanner $G=(V, E)$ obtained by the Greedy Algorithm on input $V$ and $t \geq 7.6$. By Corollary 3.4 we know that $\Delta(G) \leq 6$. Suppose that a vertex $v \in V$ that has degree 6 exists. Let $v_{1} \neq v$ be such that $d_{G}\left(v, v_{1}\right)$ is minimum. Let $v_{2}, v_{3}, \ldots, v_{6}$ be the remaining vertices connected to $v$ in clockwise order (see Fig. 2). We observe that since the algorithm examines short distances first, we have that

$$
d_{G}\left(v, v_{1}\right)=\min _{x \in V \backslash\{v\}}\{d(v, x)\}
$$

This implies that no vertex, except $v$, can be interior to the circle of center $v$ and radius $d\left(v, v_{1}\right)$. 


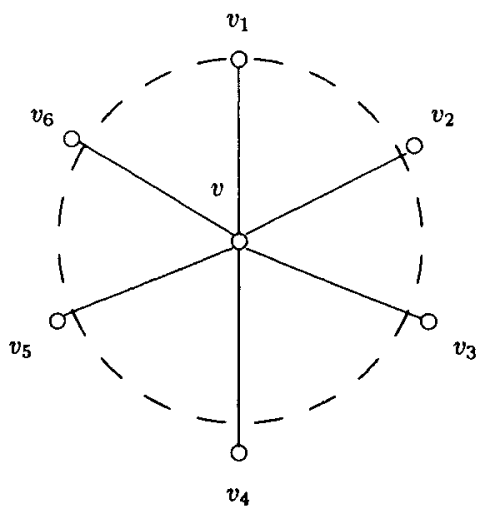

Fig. 2. A possible bad configuration.

By Proposition 2.1 the angle formed by any two edges incident with $v$ is greater than $\alpha$. Thus, clearly the angle formed by any two consecutive (clockwise order) edges is less than $\beta=2 \pi-5 \alpha$. The next proposition shows that, for sufficiently large $t$, the quotient $d\left(v, v_{i}\right) / d\left(v, v_{j}\right)$ cannot be arbitrarily large.

Proposition 4.2. Let $t \geq 13$, let $V$ be a set of points in the plane, let $\alpha=$ $2 \arcsin ((t-1) / 2 t)$, and let $\beta=2 \pi-5 \alpha$. Suppose that the Greedy Algorithm builds a t-spanner $G=(V, E)$ which has maximum degree $\Delta(G)=6$. Let $v \in V$ be a vertex with degree 6 and let $\left\{v_{1}, v_{2}, \ldots, v_{6}\right\}$ be the vertices connected to $v$ in clockwise order (see Fig. 2). Then

$$
\frac{d\left(v, v_{j}\right)}{d\left(v, v_{i}\right)}<\frac{t^{2}-1}{2 t(t \cos \beta-1)}
$$

for $|i-j|=1 \bmod 4\left(v_{i}\right.$ and $v_{j}$ are consecutive vertices $)$.

Proof. Let $r=d\left(v, v_{j}\right) / d\left(v, v_{i}\right)$. Let $\gamma=L v_{i} v v_{j}$. From Proposition 2.2 we have that

$$
\cos \gamma<\frac{1}{2 r}\left(1-\frac{1}{t^{2}}\right)+\frac{1}{t}
$$

Observing that $t \cos \beta-1>0$ for $t \geq 13$, and that $\beta \geq \gamma$, the proposition follows immediately.

We are now ready to give the desired characterization of bad configurations.

Definition 4.3. Let $V$ be a set of points in the plane, and let $t \geq 13$. Let $\alpha=$ $2 \arcsin ((t-1) / 2 t), \beta=2 \pi-5 \alpha$, and $r=\left(t^{2}-1\right) / 2 t(t \cos \beta-1)$. The 7-tuple $B$ of 
points $\left(v, v_{1}, v_{2}, \ldots, v_{6}\right)$ in $V$ is a bad configuration with respect to $t$ if the following three conditions hold:

(i) $d\left(v, v_{1}\right)=\min _{x \in V \backslash\{v\}}\{d(v, x)\}$.

(ii) $\alpha \leq \angle v_{i} v v_{j} \leq \beta$ for $|i-j|=1 \bmod 4\left(v_{i}\right.$ and $v_{j}$ are consecutive points in the bad configuration).

(iii) $d\left(v, v_{j}\right) \leq r d\left(v, v_{i}\right)$ for $|i-j|=1 \bmod 4$.

The point $v$ is the center of the bad configuration, and $d\left(v, v_{1}\right)$ is its radius, denoted by $\operatorname{radius}(B)$. The real number $t$ will be implicit when we write "bad configuration" instead of "bad configuration with respect to $t$."

Informally, the points of a bad configuration form a hexagon with a center. As $t$ increases, the angles mentioned in the definition tend to $\pi / 3$, and the quotient $d\left(v, v_{j}\right) / d\left(v, v_{i}\right)$ tends to 1 . Therefore, for large enough $t$, the hexagon will be arbitrarily close to a regular hexagon.

It should be clear that:

Proposition 4.4. If, on input $V$ and $t \geq 13$, the Greedy Algorithm outputs a graph $G=(V, E)$ with $\Delta(G)=6$, then $V$ has a bad configuration with respect to $t$.

\subsection{Removing Points from $V$}

Let $V$ be a set of points in the plane. To obtain the $\bar{t}$-spanner $G$ of $V$ with $\Delta(G) \leq 5$ we determine a set $V^{\prime} \subseteq V$ that does not have bad configurations with respect to some fixed $t^{\prime}$. The set $V^{\prime}$ and $t^{\prime}$ are the input of the Greedy Algorithm, yielding a $t^{\prime}$-spanner $G^{\prime}=\left(V^{\prime}, E^{\prime}\right)$ with $\Delta\left(G^{\prime}\right) \leq 5$. The graph $G$ is constructed from $G^{\prime}$ by considering the points in $V \backslash V^{\prime}$ and conveniently adding new edges to or removing edges from $E^{\prime}$. The algorithm that constructs $V^{\prime}$ is described below.

\section{Algorithm 4.5}

Input: a set $V$ of points in the plane and a real number $t \geq 13$.

Output: a set $V^{\prime}$ that does not have bad configurations with respect to $t$.

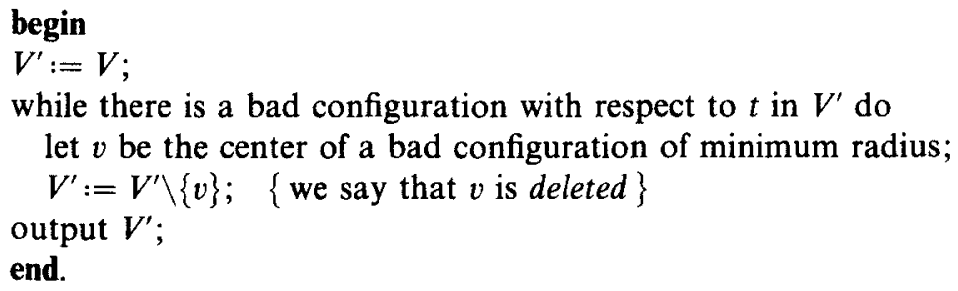

Next we prove some properties concerning the output of this algorithm. These properties are useful for constructing the spanner of $V$ from a spanner of $V^{\prime}$. In particular, the vertex $v_{1}$ of a bad configuration $B$ is the vertex that connects the 
center of $B$ to the remainder of the points in the spanner. To prove the proposition we again need to increase the value of $t$.

Proposition 4.6. If $t \geq 116$, then for any $V$ the following holds with respect to Algorithm 4.5:

(i) The sequence of minimum radii induced by the algorithm is a nondecreasing sequence.

(ii) Let $v$ be a point which is deleted by Algorithm $4.5\left(v \in V \backslash V^{\prime}\right)$. Let $B=$ $\left(v, v_{1}, v_{2}, \ldots, v_{6}\right)$ be the corresponding bad configuration. Then $\left\{v_{1}, v_{2}, v_{6}\right\} \subseteq$ $V^{\prime}$. In other words, if the center of a bad configuration is deleted the points $v_{1}, v_{2}$, and $v_{6}$ will not be deleted thereafter.

(iii) Suppose that $\left(v, v_{1}, v_{2}, \ldots, v_{6}\right)$ and $\left(v^{\prime}, v_{1}, v_{2}^{\prime}, \ldots, v_{6}^{\prime}\right)$ are two distinct bad configurations sharing the same point $v_{1}$. Also, suppose that $v$ and $v^{\prime}$ are deleted by the algorithm. Then $\angle v v_{1} v^{\prime}>2 \pi-5 \alpha=\beta$, where $\alpha=2 \arcsin ((t-1) / 2 t)$.

Proof. Let $T$ be the set of points corresponding to $V^{\prime}$ in an arbitrary step of Algorithm 4.5. Let $v$ be the center of a bad configuration $B=\left(v, v_{1}, \ldots, v_{6}\right)$ of minimum radius $d\left(v, v_{1}\right)$ in $T$. Suppose that $v$ is about to be deleted. Let $B^{\prime}$ be a bad configuration in $T \backslash\{v\}$. We are going to show that the radius of $B^{\prime}$ is at least $\operatorname{radius}(B)=d\left(v, v_{1}\right)$.

Since the algorithm chose the center $v$ to be deleted, it follows that if $B^{\prime}$ is a bad configuration in $T$, then radius $\left(B^{\prime}\right) \geq \operatorname{radius}(B)$, and we are done.

So, suppose that $B^{\prime}$ is not a bad configuration in $T$ and $B^{\prime}$ becomes one in $T \backslash\{v\}$. Since the removal of $v$ from $T$ caused $B^{\prime}$ to become a bad configuration, condition (i) of Definition 4.3 has changed: the deletion of points cannot make Definitions 4.3(ii) or 4.3(iii) true. Let $w$ be the center of $B^{\prime}$. By the previous observation we have that $\min \{d(w, x) \mid x \in T \backslash\{v, w\}\}>\min \{d(w, x) \mid x \in T \backslash\{w\}\}$. Thus, $v$ is the unique point nearest to $w$ in $T$ and $d(v, w)<\operatorname{radius}\left(B^{\prime}\right)$. By the definition of a bad configuration (item (i)) radius $(B) \leq d(v, w)$. Combining the last two inequalities we finish the proof of (i).

Throughout the remainder of the proof we consider the following numerical values and variables definitions. The meaning of some variables and expressions will be clear later.

The numerical values follow from the assumption that $t \geq 116$.

$$
\begin{gathered}
1.037<\alpha=2 \arcsin \frac{t-1}{2 t}<\frac{\pi}{3}<\beta=2 \pi-5 \alpha<1.097, \quad \cos \left(\frac{\beta}{2}\right)>0.853, \\
r=\frac{t^{2}-1}{2 t(t \cos \beta-1)}<1.117, \quad s=\sqrt{1+r(r-2 \cos \beta)}<1.109, \\
r^{2}-1<0.247, \quad \frac{r^{6} s^{2}+r^{2}-1}{2 r^{3} s}<0.852, \quad 1-\cos \alpha>0.491,
\end{gathered}
$$




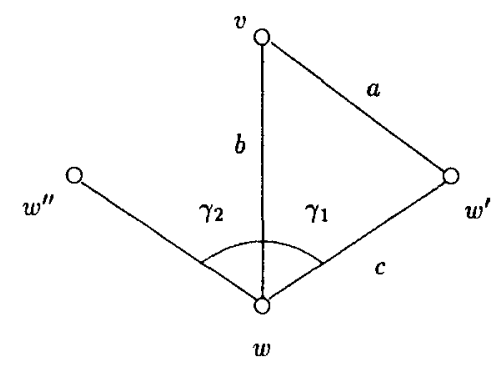

Fig. 3. Illustration for Proposition 4.6(ii).

$$
\begin{gathered}
\frac{\sqrt{3}}{2}>\sin \alpha>0.861, \quad \frac{r^{2}-1}{2}<0.124, \quad \pi-\alpha<2.105, \\
r-\cos \alpha<0.609, \quad \frac{\pi-3 \beta}{2}>-0.075 .
\end{gathered}
$$

Now we prove (ii). Actually, we prove the slightly stronger statement: after the deletion of $v$, no point in the set $\left\{v_{1}, v_{2}, v_{6}\right\}$ can become a center of a bad configuration. By contradiction, suppose that after the deletion of $v, w$ is the first point in the set $\left\{v_{1}, v_{2}, v_{6}\right\}$ that becomes a center of a bad configuration $B^{\prime}$. Let $T$ be the set of points corresponding to $V^{\prime}$ in a step of the algorithm in which $B^{\prime}$ is a bad configuration.

Consider the configuration shown in Fig. 3, where $w^{\prime}$ and $w^{\prime \prime}$ are two consecutive points of $B^{\prime}$ such that $w^{\prime}$ and $w^{\prime \prime}$ are in different semiplanes defined by the line containing $v$ and $w$. Let $a=d\left(v, w^{\prime}\right), b=d(v, w), c=d\left(w, w^{\prime}\right) . \gamma_{1}=\angle v w w^{\prime}, \gamma_{2}=$ $\angle v w w^{\prime \prime}, R=\operatorname{radius}(B)$, and $R^{\prime}=\operatorname{radius}\left(B^{\prime}\right)$. We prove that $\gamma_{1}+\gamma_{2}>\beta$. This contradicts item (ii) of Definition 4.3 , showing that $B^{\prime}$ is not a bad configuration. Without loss of generality suppose that $\gamma_{1} \leq \gamma_{2}$.

We have that $a^{2}=b^{2}+c^{2}-2 b c \cos \gamma_{1}$ or

$$
\cos \gamma_{1}=\frac{b^{2}+c^{2}-a^{2}}{2 b c} .
$$

Since $w \in\left\{v_{1}, v_{2}, v_{6}\right\}$ it follows from Definition 4.3(iii) that

$$
b \leq \max \left\{d\left(v, v_{1}\right), d\left(v, v_{2}\right), d\left(v, v_{6}\right)\right\} \leq r R .
$$

By Definition 4.3(i) we have that $a \geq R$ and $b \geq R$. Using these inequalities in (4) we obtain

$$
\cos \gamma_{1} \leq \frac{c^{2}+r^{2} R^{2}-R^{2}}{2 R c}=\frac{c^{2}+R^{2}\left(r^{2}-1\right)}{2 R c}
$$


We now inspect two cases:

Case 1: $c^{2} \leq R^{2}\left(r^{2}-1\right)$. From (5) we obtain

$$
\cos \gamma_{1} \leq \frac{2 R^{2}\left(r^{2}-1\right)}{2 R c}=\frac{R\left(r^{2}-1\right)}{c}
$$

By (i) $\operatorname{radius}\left(B^{\prime}\right) \geq \operatorname{radius}(B)$, and by the definition of the radius we have that $c \geq R^{\prime}$, implying that $c \geq R$. Thus,

$$
\cos \gamma_{1} \leq \frac{R\left(r^{2}-1\right)}{R}=r^{2}-1<\cos \left(\frac{\beta}{2}\right)
$$

To obtain the last inequality we have used the numerical values calculated in (3). Thus, $\gamma_{1}>\beta / 2$, implying that $\gamma_{1}+\gamma_{2}>\beta$. As observed before, this contradicts Definition 4.3(ii).

Case 2: $c^{2} \geq R^{2}\left(r^{2}-1\right)$. We begin determining an upper bound for $c$. Since the points $v_{1}, v_{2}, v_{6}$ are in $T$, and $w$ is one of these points, we have by Definition 4.3(i) that $\operatorname{radius}\left(B^{\prime}\right) \leq \max \left\{d\left(v_{1}, v_{2}\right), d\left(v_{1}, v_{6}\right)\right\}$. Without loss of generality, suppose that $d\left(v_{1}, v_{2}\right) \geq d\left(v_{1}, v_{6}\right)$. Let $\gamma=\angle v_{1} v v_{2}$. Then

$$
\begin{aligned}
R^{\prime 2} & \leq d^{2}\left(v_{1}, v_{2}\right)=d^{2}\left(v, v_{1}\right)+d^{2}\left(v, v_{2}\right)-2 d\left(v, v_{1}\right) d\left(v, v_{2}\right) \cos \gamma \\
& =R^{2}+d^{2}\left(v, v_{2}\right)-2 R d\left(v, v_{2}\right) \cos \gamma
\end{aligned}
$$

By Definition 4.3(iii) $d\left(v, v_{2}\right) \leq r R$. Using that $\gamma \leq \beta$, and that

$$
d\left(v, v_{2}\right)-2 R \cos \beta \geq 0
$$

we obtain

$$
\begin{aligned}
R^{\prime 2} & \leq R^{2}+d^{2}\left(v, v_{2}\right)-2 R d\left(v, v_{2}\right) \cos \beta \leq R^{2}+d\left(v, v_{2}\right)\left(d\left(v, v_{2}\right)-2 R \cos \beta\right) \\
& \leq R^{2}+r R(r R-2 R \cos \beta) \leq R^{2}(1+r(r-2 \cos \beta)),
\end{aligned}
$$

or

$$
R^{\prime} \leq s R, \quad \text { where } s=\sqrt{1+r(r-2 \cos \beta)}
$$

By item (iii) of Definition 4.3 of a bad configuration we have that $c \leq r^{3} R^{\prime}$. Using this inequality in (7) we have that $c \leq r^{3} s R$. Then, from (5) and the assumption that $c^{2} \geq R^{2}\left(r^{2}-1\right)$, we obtain

$$
\cos \gamma_{1} \leq \frac{r^{6} s^{2} R^{2}+R^{2}\left(r^{2}-1\right)}{2 r^{3} s R^{2}}=\frac{r^{6} s^{2}+r^{2}-1}{2 r^{3} s}<\cos \left(\frac{\beta}{2}\right) \text {. }
$$


To obtain the last inequality we have used the numerical values calculated in (3). Thus, $\gamma_{1}>\beta / 2$, implying that $\gamma_{1}+\gamma_{2}>\beta$. Again, this contradicts Definition 4.3(ii), finishing the proof of (ii).

We now prove (iii). Let $R=d\left(v, v_{1}\right)$ and $R^{\prime}=d\left(v^{\prime}, v_{1}\right)$. Without loss of generality suppose that $v$ was deleted before $v^{\prime}$ by the algorithm. This implies that

$$
R \leq R^{\prime}
$$

We may also suppose that $v^{\prime}$ and $v_{2}$ are in the same semiplane defined by the line containing $v v_{1}$ (if this is not true, interchange the roles of $v_{2}$ and $v_{6}$ in the proof). Thus, we assume that

$$
0 \leq \gamma_{2} \leq \pi
$$

where $\gamma_{2}=\angle v_{1} v v^{\prime}$.

Let $a=d\left(v^{\prime}, v_{2}\right), \quad b=d\left(v, v_{2}\right), \quad c=d\left(v, v^{\prime}\right), \quad \gamma_{1}=\angle v v_{1} v^{\prime}, \quad \gamma_{3}=\angle v v^{\prime} v_{1}, \quad$ and $\theta= \pm \angle v^{\prime} v v_{2}$. Figure 4 illustrates these measures. The sign of $\theta$ is positive if the points $v_{1}, v_{2}$, and $v^{\prime}$ appear in clockwise order considering $v$ as a center (Fig. 4(a)). The sign of $\theta$ is negative otherwise (Fig. $4(\mathrm{~b})$ ).

We want to prove that $\gamma_{1}>\beta$. By contradiction, suppose that

$$
\gamma_{1} \leq \beta
$$

We have that

$$
\begin{gathered}
a^{2}=b^{2}+c^{2}-2 b c \cos \theta, \\
R^{\prime 2}=R^{2}+c^{2}-2 R c \cos \gamma_{2} .
\end{gathered}
$$

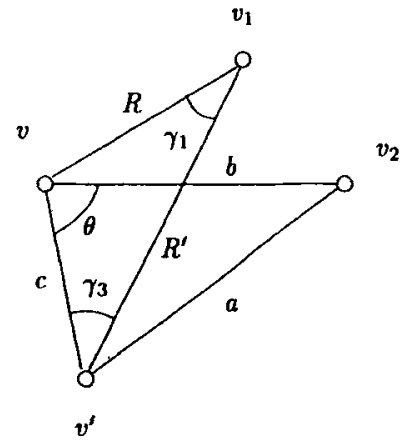

(a)

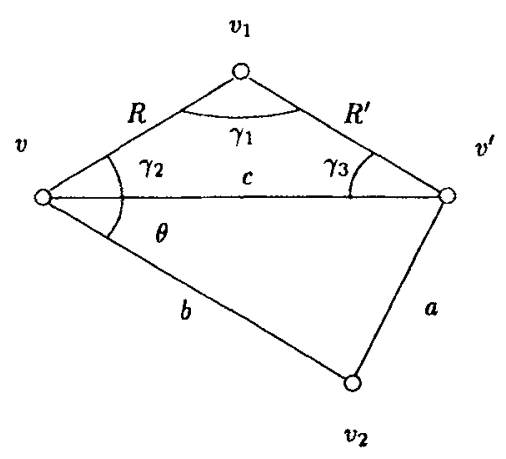

(b)

Fig. 4. Illustration for Proposition 4.6(iii). 
By (ii) we have that when the point $v^{\prime}$ was deleted by the algorithm, the point $v_{2}$ was in the set $V^{\prime}$. Thus, by the definition of radius of a bad configuration we have that $a \geq R^{\prime}$. Thus,

$$
a^{2}=b^{2}+c^{2}-2 b c \cos \theta \geq R^{\prime 2}=R^{2}+c^{2}-2 R c \cos \gamma_{2}
$$

or

$$
\frac{b^{2}-R^{2}}{2 c} \geq b \cos \theta-R \cos \gamma_{2}
$$

By the definition of a bad configuration we have that $c \geq R$ and $b \leq r R$. Thus,

$$
\frac{R r^{2}-R}{2} \geq b \cos \theta-R \cos \gamma_{2}
$$

By the definition of a bad configuration we have that $\alpha \leq \angle v_{1} v v_{2}=\gamma_{2}-\theta$. Using these inequalities we obtain that $\gamma_{2} \geq \theta+\alpha$. Thus,

$$
\frac{R r^{2}-R}{2} \geq b \cos \theta-R \cos (\theta+\alpha)=(b-R \cos \alpha) \cos \theta+R \sin \alpha \sin \theta
$$

We have also used above that, by (9), $\gamma_{2} \leq \pi$.

We now inspect two cases:

Case 1: $\theta \leq \pi / 2$. Since, by (8), $R \leq R^{\prime}$, we have that $\gamma_{2} \geq \gamma_{3}$. Thus, using (10), $\pi=\gamma_{1}+\gamma_{2}+\gamma_{3} \leq \beta+2 \gamma_{2}$ or $\gamma_{2} \geq(\pi-\beta) / 2$. By the definition of a bad configuration we have that $\angle v_{1} v v_{2}=\gamma_{2}-\theta \leq \beta$. Using these inequalities we obtain that

$$
\theta \geq \frac{\pi-3 \beta}{2}>-0.075
$$

In the last inequality we have used the numerical values calculated in (3).

Also, by the definition of radius of a bad configuration, $b \geq R$. Thus, for $\pi / 2 \geq \theta>-0.075$, we have from (11) that

$$
\frac{R r^{2}-R}{2} \geq(R-R \cos \alpha) \cos \theta+R \sin \alpha \sin \theta
$$

or

$$
\frac{r^{2}-1}{2} \geq(1-\cos \alpha) \cos \theta+\sin \alpha \sin \theta>0.491 \cos \theta+\sin \alpha \sin \theta
$$

In the last inequality we have used the numerical values calculated in (3). 
For $\pi / 2 \geq \theta \geq-0.075$, the minimum of $0.491 \cos \theta+\sin \alpha \sin \theta$ is attained for $\theta=-0.075$. Thus, $0.419 \cos \theta+\sin \alpha \sin \theta>0.419 \cos \theta+\sqrt{3} / 2 \sin \theta>0.42$. On the other hand, by (3) we have that $0.124>\left(r^{2}-1\right) / 2$. These values contradict inequality (13).

Case 2: $\theta>\pi / 2$. By the definition of a bad configuration we have that $\alpha \leq$ $\angle v_{1} v v_{2}=\gamma_{2}-\theta$. Using this inequality and (9) we obtain that

$$
\theta \leq \gamma_{2}-\alpha \leq \pi-\alpha
$$

Considering that by the definition of a bad configuration $b \leq R r$, and that $\pi / 2<\theta \leq \pi-\alpha$ we obtain from (11) that

$$
\frac{R r^{2}-R}{2} \geq(r R-R \cos \alpha) \cos \theta+R \sin \alpha \sin \theta
$$

or

$$
\frac{r^{2}-1}{2} \geq(r-\cos \alpha) \cos \theta+\sin \alpha \sin \theta>0.609 \cos \theta+0.861 \sin \theta
$$

In the last inequality we have used the numerical values calculated in (3).

For $\pi / 2<\theta \leq \pi-\alpha$, the minimum of $0.609 \cos \theta+0.861 \sin \theta$ is attained for $\theta=\pi-\alpha<2.105$. Thus, $0.609 \cos \theta+0.861 \sin \theta>0.43$. On the other hand, by (3) we have that $0.124>\left(r^{2}-1\right) / 2$. These values contradict inequality (15).

\subsection{The Algorithm for $\Delta \leq 5$}

The next algorithm uses Algorithm 4.5 and the Greedy Algorithm to construct $G$ with $\Delta(G) \leq 5$.

\section{Algorithm 4.7}

Input: a set $V$ of points in the plane and a real number $t \geq 116$.

Output: a graph $G=(V, E)$.

\section{begin}

Let $V^{\prime}$ be the output of Algorithm 4.5 on input $V$ and $t$;

Let $G^{\prime}=\left(V^{\prime}, E^{\prime}\right)$ be the output of the Greedy Algorithm on input $V^{\prime}$ and $t$;

$E:=E^{\prime}$;

$\alpha:=2 \arcsin ((t-1) / 2 t)$;

$\beta:=2 \pi-5 \alpha$;

for each $v \in V \backslash V^{\prime}$ do 
let $\left(v, v_{1}, v_{2}, \ldots, v_{6}\right)$ be the bad configuration that caused $v$ to be deleted by Algorithm 4.5;

if $v_{1} w \in E^{\prime}$ exists such that $\angle v v_{1} w \leq \beta$

then mark $v_{1} w$ (we say that $v_{1} w$ is marked near $v_{1}$ and that $v$ caused $v_{1} w$ to be marked near $v_{1}$ );

else $E:=E \cup\left\{v v_{1}\right\}$

while a marked edge $x y \in E$ exists do $\quad\{$ Loop 2$\}$

let $X=\left\{x_{1}, x_{2}\right\} \subseteq V$ be centers of bad configurations that caused $x y$ to be marked near $x$;

let $Y=\left\{y_{1}, y_{2}\right\} \subseteq V$ be centers of bad configurations that caused $x y$ to be marked near $y$;

if $|X|=1(|Y|=1)$ then consider $x_{1}=x_{2}\left(y_{1}=y_{2}\right)$;

if $X=\varnothing(Y=\varnothing)$ then consider $x=x_{1}=x_{2}\left(y=y_{1}=y_{2}\right)$;

relabel the points in $X$ and $Y$ to make $d\left(x_{1}, x\right) \leq d\left(x_{2}, x\right)$ and $d\left(y_{1}, y\right) \leq d\left(y_{2}, y\right)$ \{see Fig. 5(a)\}

$E:=(E \backslash\{x y\}) \cup\left\{x_{1} x, x_{1} x_{2}, y_{1} y, y_{1} y_{2}, x_{1} y_{1}\right\} ;$

output $G=(V, E)$;

end.

Proposition 4.8. Let $G=(V, E)$ be the output of Algorithm 4.7 on input $V$ and $t_{1}$, where $V$ is a set of points in the plane and $t_{1}=116$. Then the following hold:

(i) An edge $x y \in E^{\prime}$ is marked near $x$ at most twice.

(ii) $\Delta(G) \leq 5$.

(iii) If $v$ causes the edge $x y$ to be marked near $x$, then $d_{G}(x, v)<t_{2} d(x, v)$, where $t_{2}=2.78$.

(iv) $d_{G}(x, y)<t_{1} t_{3} d(x, y)$ for each pair $x, y \in V^{\prime}$, where $t_{3}=4.15$.

(v) $d_{G}(x, y)<\left(3 t_{1} t_{3}+2 t_{2}\right) d(x, y)<2000 d(x, y)$ for each pair $x, y \in V$.

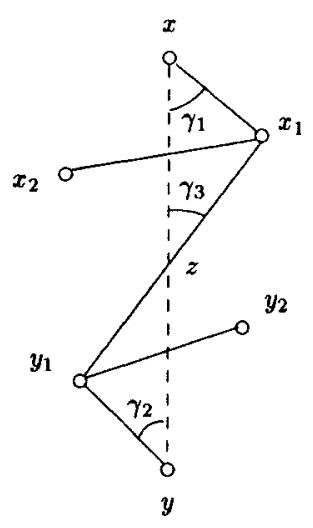

(a)

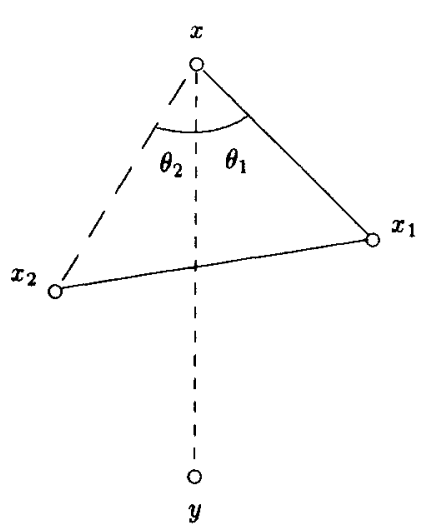

(b)

Fig. 5. Illustration for Algorithm 4.7 and Proposition 4.8. 
Proof. Let $\alpha=2 \arcsin ((t-1) / 2 t)$ and $\beta=2 \pi-5 \alpha=62.7$. Taking into consideration that if a point $v$ causes the edge $x y$ to be marked near $x$, then $\angle v x y \leq \beta$, item (i) is an easy consequence of Proposition 4.6(iii). This property was used in the algorithm to assume that $|X|,|Y| \leq 2$.

To prove (ii) observe that:

(1) Whenever an edge $v v_{1}$ is added to $E$ during the execution of Loop 1 , then $\angle v v_{1} w>\beta$ for each $v_{1} w \in E$ such that $v \neq w$. This is true by construction if $v_{1} w \in E^{\prime}$, and by Proposition 4.6(iii) if $v_{1} w \in E \backslash E^{\prime}$. Thus, adding the facts that $\Delta\left(G^{\prime}\right) \leq 5$ and that $G^{\prime}$ satisfies Proposition 2.1, we conclude that when the algorithm exits Loop 1 it is still true that $\Delta(G) \leq 5$.

(2) In Loop 2 the degree in $G$ of vertices in $V^{\prime}$ stays the same.

(3) The degree in $G$ of a vertex $v \in V \backslash V^{\prime}$ is at most 3.

We now prove (iii). If $x y$ is marked near $x$ exactly once, then $d_{G}(x, v)=d(x, v)$. So, suppose that two points, $x_{1}$ and $x_{2}$, caused $x y$ to be marked near $x$ (by (i), at most two such vertices exist). Consider Fig. $5(\mathrm{~b})$. Since the edge was marked in Loop 1 we have that $\theta_{1} \leq \beta, \theta_{2} \leq \beta$. By Loop 2 we have that $d\left(x_{1}, x\right) \leq d\left(x_{2}, x\right)$, $x_{2} x_{1}, x_{1} x \in E$. So, if $v=x_{1}$ there is nothing to prove. So, we assume that $v=x_{2}$. From the above observations we have that

$$
d_{G}\left(x, x_{2}\right) \leq d_{G}\left(x_{2}, x_{1}\right)+d_{G}\left(x_{1}, x\right)=d\left(x_{2}, x_{1}\right)+d\left(x_{1}, x\right)
$$

On the other hand,

$$
\begin{aligned}
d^{2}\left(x_{1}, x_{2}\right) & =d^{2}\left(x, x_{2}\right)+d^{2}\left(x, x_{1}\right)-2 \cos \left(\theta_{2}+\theta_{1}\right) d\left(x, x_{2}\right) d\left(x, x_{1}\right) \\
& \leq d^{2}\left(x, x_{2}\right)+d^{2}\left(x, x_{1}\right)-2 \cos (2 \beta) d\left(x, x_{2}\right) d\left(x, x_{1}\right) \\
& \leq 2 d^{2}\left(x, x_{2}\right)(1-\cos (2 \beta)) \\
& =4 d^{2}\left(x, x_{2}\right) \sin ^{2} \beta
\end{aligned}
$$

Using the previous two inequalities we obtain

$$
d_{G}(v, x) \leq 2 d(v, x) \sin \beta+d\left(x_{1}, x\right) \leq(2 \sin \beta+1) d(v, x)<2.79 d(v, x)=t_{2} d(v, x) .
$$

We now prove (iv).

Case 1: $x y \in E^{\prime}$. If $x y$ is never marked near $x(y)$ by Algorithm 4.7, then let $x_{1}=x$ $\left(y_{1}=y\right)$. Otherwise, let $x_{1}\left(y_{1}\right)$ be such that $d\left(x_{1}, x\right)\left(d\left(y_{1}, y\right)\right)$ is minimum for the points which marked $x y$ near $x(y)$.

By construction we have that

$$
d_{G}(x, y) \leq d\left(x, x_{1}\right)+d\left(x_{1}, y_{1}\right)+d\left(y_{1}, y\right)
$$

If $x_{1}$ and $y_{1}$ are in the same semiplane defined by the line containing $x y$, we may switch $x_{1}$ (or $x_{2}$ ) to the other semiplane without changing the absolute value of $\gamma_{1}$. In this modified configuration the distance in the graph between $x$ and $y$ is 
larger than the original distance. Thus, we assume the worst case, i.e., $x_{1}$ and $y_{1}$ are not in the same semiplane defined by the line containing $x y$ (see Fig. 5(a)).

Since the points $x, y$ are in $V^{\prime}$, at the time that $x_{1}$ was deleted the vertex $y$ was in the set $V^{\prime}$. This implies by the definition of a bad configuration that $d\left(x_{1}, x\right) \leq$ $d\left(x_{1}, y\right)$. Similarly, it is true that $d\left(y_{1}, y\right) \leq d\left(y_{1}, x\right)$. This implies that

$$
\gamma_{3} \leq \frac{\pi}{2}
$$

As in the proof of (iii) we have that

$$
\gamma_{1} \leq \beta \quad \text { and } \quad \gamma_{2} \leq \beta
$$

Let $z$ the point defined by the intersection of the lines $x y$ and $x_{1} y_{1}$. From (16) we obtain

$$
d_{G}(x, y) \leq d\left(x, x_{1}\right)+d\left(x_{1}, z\right)+d\left(z, y_{1}\right)+d\left(y_{1}, y\right) \text {. }
$$

Observe that $d(x, z) \sin \gamma_{1}=d\left(x_{1}, z\right) \sin \left(\pi-\gamma_{1}-\gamma_{3}\right)$ and

$$
d(x, z) \sin \gamma_{3}=d\left(x, x_{1}\right) \sin \left(\pi-\gamma_{1}-\gamma_{3}\right)
$$

Thus,

$$
d\left(x, x_{1}\right)+d\left(x_{1}, z\right)=d(x, z) \frac{\sin \gamma_{1}+\sin \gamma_{3}}{\sin \left(\gamma_{1}+\gamma_{3}\right)}
$$

The maximum of the function $\left(\sin \gamma_{1}+\sin \gamma_{3}\right) / \sin \left(\gamma_{1}+\gamma_{3}\right)$ subject to (17) and (18) occurs when $\gamma_{3}=\pi / 2$ and $\gamma_{1}=\beta$, implying that

$$
d\left(x, x_{1}\right)+d\left(x_{1}, z\right) \leq d(x, z) \frac{\sin \beta+1}{\cos \beta}
$$

Similarly

$$
d\left(y, y_{1}\right)+d\left(y_{1}, z\right) \leq d(y, z) \frac{\sin \beta+1}{\cos \beta} .
$$

Using the last two inequalities in (19) we obtain

$$
\begin{aligned}
d_{G}(x, y) & \leq d(x, z) \frac{\sin \beta+1}{\cos \beta}+d(y, z) \frac{\sin \beta+1}{\cos \beta}=d(x, y) \frac{\sin \beta+1}{\cos \beta}<4.18 d(x, y) \\
& =t_{3} d(x, y) .
\end{aligned}
$$


Case 2: $x y \notin E^{\prime}$. Then, since $G^{\prime}$ is the output of the Greedy Algorithm, there is a path $P$ in $G^{\prime}$ that has total length $\leq t_{1} d(x, y)$. By the previous case, for each edge $v w$ in $P$ there is a path in $G$ such that $d_{G}(v, w)<t_{3} d(v, w)$. Therefore, the quotient between the length of an $x y$-path in $G$ and the distance between $x$ and $y$ is less than $t_{1} t_{3}$.

We now prove (v). If $x(y) \in V^{\prime}$, then let $v_{1}=x\left(v_{1}^{\prime}=y\right)$. Otherwise, let $v_{1}\left(v_{1}^{\prime}\right)$ be the point nearest to $x(y)$ in the bad configuration that caused $x(y)$ to be deleted. Either way we have that $v_{1}, v_{1}^{\prime} \in V^{\prime}$ (in the last case, this follows from Proposition 4.6(ii)). It follows from (iv) that

$$
d_{G}\left(v_{1}, v_{1}^{\prime}\right)<t_{1} t_{3} d\left(v_{1}, v_{1}^{\prime}\right)
$$

It follows from (iii) that

$$
d_{G}\left(x, v_{1}\right)<t_{2} d\left(x, v_{1}\right) \text { and } d_{G}\left(y, v_{1}^{\prime}\right)<t_{2} d\left(y, v_{1}^{\prime}\right)
$$

It follows from Definition 4.3(i) that

$$
d\left(x, v_{1}\right) \leq d(x, y)
$$

It also follows from Definition 4.3(i) that $d\left(y, v_{1}^{\prime}\right) \leq d\left(y, v_{1}\right)$. Using this inequality, the triangle inequality property, and (22) it follows that

$$
d\left(y, v_{1}^{\prime}\right) \leq d\left(y, v_{1}\right) \leq d(x, y)+d\left(x, v_{1}\right) \leq 2 d(x, y) .
$$

Using (20)-(23) and the triangle inequality property we have that

$$
\begin{aligned}
d_{G}(x, y) & \leq d_{G}\left(x, v_{1}\right)+d_{G}\left(v_{1}, v_{1}^{\prime}\right)+d_{G}\left(v_{1}^{\prime}, y\right) \\
& \leq t_{2} d\left(x, v_{1}\right)+t_{1} t_{3} d\left(v_{1}, v_{1}^{\prime}\right)+t_{2} d\left(v_{1}^{\prime}, y\right) \\
& <t_{2} d(x, y)+t_{1} t_{3}\left(d\left(v_{1}, x\right)+d(x, y)+d\left(y, v_{1}^{\prime}\right)\right)+2 t_{2} d(x, y) \\
& \leq t_{2} d(x, y)+4 t_{1} t_{3} d(x, y)+2 t_{2} d(x, y) \\
& \leq\left(4 t_{1} t_{3}+3 t_{2}\right) d(x, y)<2000 d(x, y)
\end{aligned}
$$

Remark 4.9. Many bounds in the proofs are not the best possible. Since we could not obtain the final constant less than a 100 , we would rather simplify the proof than obtain a tighter bound.

\section{Open Problems}

1. It remains open whether or not $\Delta=4$ or 3 suffices to approximate planar distances within a constant factor. Dobkin et al. [5] observed that for $\Delta=2$ such a result is impossible. 
2. Find better upper bounds on the number of edges sufficient to approximate $d$-dimensional Euclidean distances.

3. Also considering the $d$-dimensional Euclidean space, nontrivial lower bounds are not known either for $\Delta$ or for the number of edges necessary to the approximation.

4. Consider that we are allowed to, at our convenience, add extra points to the given set of points. (A concept similar to Steiner trees.) Would we be able to prove better upper bounds on the number of edges sufficient to approximate Euclidean distances?

5. What is the complexity of the following problem:

Instance: $V \subset \mathbb{R}^{d}, t, m \geq 1$.

Question: Does $V$ have a $t$-spanner with at most $m$ edges?

We observe that Peleg and Schäffer [11] have proved that this problem is NP-complete in the case that $V$ is from an arbitrary metric space.

6. Find a polynomial-time algorithm $\mathscr{A}$ to build $t$-spanners such that $\mathscr{A}$ has "performance guarantees," i.e., on every input $V \subset \mathbb{R}^{d}, \mathscr{A}$ builds a $t$-spanner $G=(V, E)$ such that $|E| \leq c \cdot e_{\text {opt }}$, where $c$ is a constant and $e_{\mathrm{opt}}$ is the minimum number of edges over all $t$-spanners of $V$.

Remark 5.1. After the submission of this paper, the author received a preprint by Salowe [15] who proved that, for any fixed dimension $d$, a constant $t$ exists such that any set of points in the $d$-dimensional Euclidean space has a $t$-spanner with maximum degree 4.

\section{References}

1. Althöfer, I.; Das, G.; Dobkin, D; Joseph, D., Generating sparse spanners for weighted graphs, Proc. Second Scandinavian Workshop on Algorithm Theory, 1990, pp. 26-37.

2. Althöfer, I.; Das, G.; Dobkin, D; Joseph, D.; Soares, J., On sparse spanners of weighted graphs, Discrete Comput. Geom. 9 (1993), 81-100.

3. Chew, L. P., There is a planar graph almost as good as the complete graph, Proc. Second Symposium on Computational Geometry, 1986, pp. 169-177.

4. Conway, J. H; Sloane, N. J. A., Sphere Packings, Lattices, and Groups, Springer-Verlag, New York, 1988.

5. Dobkin, P. D.; Friedman, S. J.; Supowit, K. J., Delaunay graphs are almost as good as complete graphs, Discrete Comput. Geom. 5 (1990), 399-407.

6. Kabatiansky, G. A.; Levenshtein, V. I., Bounds for packings on a sphere and in space, Problems Inform. Transmission 14 (1978), 1-17.

7. Keil, J. M., Approximating the complete Euclidean graph, Proc. First Scandinavian Workshop on Algorithm Theory, 1988, pp. 208-213.

8. Keil, J. M.; Gutwin, C. A., Classes of graphs which approximate the complete Euclidean graph, Discrete Comput. Geom. 7 (1992), 13-28.

9. Levcopoulos, C.; Lingas, A., There are planar graphs almost as good as the complete graphs and as short as the minimum spanning trees, Proc. Symposium on Optimal Algorithms, 1989, pp.9-13.

10. Nisan, N., Personal communication, 1990.

11. Peleg, D.; Schäffer. A., Graphs spanners, J. Graph Theory, 13 (1989), 99-116.

12. Rankin, R. A., The closest packing of spherical caps in $n$ dimensions, Proc. Glasgow Math. Assoc. 2 (1955), 139-144. 
13. Ruppert, J.; Seidel, R., Approximating the $d$-dimensional complete Euclidean graph, Proc. Canadian Conference on Computational Geometry, 1991, pp. 207-210.

14. Salowe, J. S., Construction of multidimensional spanner graphs, with applications to minimum spanning trees, Proc. Seventh Symposium on Computational Geometry, 1991, pp. 256-261.

15. Salowe, J., On Euclidean spanners graphs with small degree, Proc. 8th ACM Symposium on Computational Geometry, 1992, pp. 192-201.

16. Soares, J., Approximating metrics by graphs, Manuscript, 1990.

17. Vaidya, P. M., An $O(n \log n)$ algorithm for the all-nearest-neighbors problem, Discrete Comput. Geom. 4 (1989), 101-115.

18. Vaidya, P. M., A sparse graph almost as good as the complete graph on points in $k$ dimensions, Discrete Comput. Geom. 6 (1991), 369-381.

Received March 6, 1992, and in revised form July 14, 1993 